\title{
Selected Harmonic Elimination Pulse Width Modulation Based on the Homotopy Algorithm
}

\author{
Xiaoci Wang ${ }^{1}$ \\ ${ }^{1}$ School of Electrical and Electric Engineering, North China Electric Power University, Beijing, \\ 102206
}

Keywords: SHEPWM; the homotopyalrorithm; cascade multi-level inverter

\begin{abstract}
SHEPWM, a method to eliminate the selected low order harmonics by optimizing switching time sequence, has been concerned greatly because of its advantages such as high waveform quality, high efficiency, full utilization of DC side voltage and small size of DC side filter. In this paper, amathematic model based on thehomotopy algorithm is proposed to calculate the optimal switching time. The method is applied to three-level voltage inverters toshow the operations in detail. And to get more accurate value of switching time, the model is optimized with combining with Newton's method.
\end{abstract}

\section{Introduction}

In recent years, a novelcascade multi-level inverter topology enters the public arena for its great output capacity, high power application, high switching frequency and harmonic performance of output voltage and current. It has been used widely in high power occasions such as Flexible AC Transmission Systems (FACTS). Selected Harmonic Elimination Pulse Width Modulation (SHEPWM) is an important technique to control the cascade multi-level inverter topology by selecting the optimal switching time to eliminate the selected low-frequency harmonics and improve the waveform quality.

\section{Model of Multi-level Inverter}

The cascade multi-level inverter is formed by several basic inverter unit (such as the $\mathrm{H}$-bridge inverter) connected through a series of single-phase or three-phase inverter, shown in figure 1. Shown in figure 2, output voltage $v_{a c}$ of the cascade multi-level inverter is superposed by output voltage of each inverter unit. With the increase in the number of the inverter cascade, the stepped SPWM waveforms is more approximate to sine. 


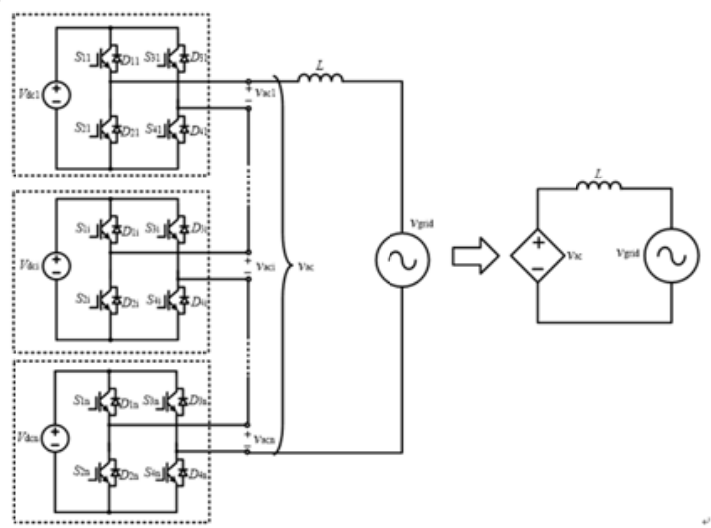

Figure 1 Grid system structure and equivalent circuit of cascade H-bridge inverter

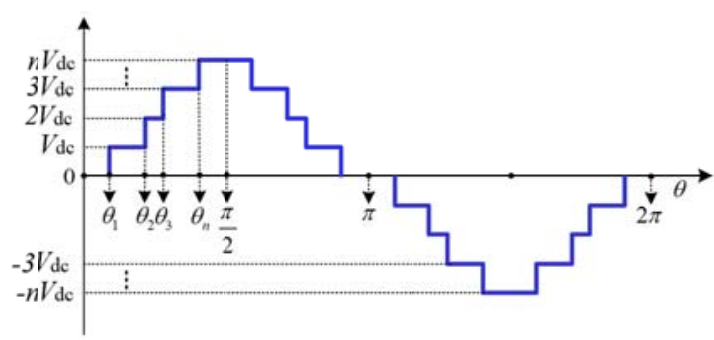

Figure 2 output voltage of the stepped SPWM waveforms

For the ith H-bridge inverter unit in the cascade inverters systems, output is 0 level when $\left(S_{1 i}, S_{3 i}\right)$ or $\left(S_{2 i}, S_{4 i}\right)$ close. Output is 1 level when $\left(S_{1 i}, S_{4 i}\right)$ close, namely the output voltage $v_{a c i}=V_{d c i}$; Output is -1 level when $\left(S_{2 i}, S_{3 i}\right)$ close, namely the output voltage $v_{a c i}=-V_{d c i}$. The overall output levels of H-bridge inverter can be $(2 n+1)$.

\section{Nonlinear Equations}

The output of each H-bridge module is half-wave symmetric and 1/4-wave symmetric. Decomposite the wave with Fourier series, the amplitude of s-th odd harmonics is expressed as follows:

$$
V_{s}=\frac{4 V_{d c}}{s \pi} \sum_{s=1,3,5, \cdots}^{\infty}\left[\cos \left(s \theta_{1}\right)+\cos \left(s \theta_{2}\right)+\cdots+\cos \left(s \theta_{n}\right)\right]
$$

Where $0 \leq \theta_{1}<\theta_{2}<\cdots<\theta_{n} \leq \frac{\pi}{2}$.

For the cascade converter system that has $\mathrm{n} H$-bridge, the number of specific harmonic that can be eliminated is (n-1) in meet the expectation of fundamental voltage amplitude conditions. When a set of $\theta_{i}(i=1,2, \cdots, n)$ is numerical suitable, the fundamental component amplitude of the output voltage can be $V_{1 m}$, and low-order harmonic can be eliminated.(The multiple of 3 times harmonic component does not take into account in symmetrical three-phase systems.)

Define modulation ratio of amplitude is $\mathrm{m}=\frac{V_{1 m}}{n \cdot V_{d c}}$. In accordance with the conditions above, the nonlinear equations are as follow, 


$$
\left\{\begin{aligned}
& \cos \left(\theta_{1}\right)+\cos \left(\theta_{2}\right)+\cdots+\cos \left(\theta_{n}\right)=\frac{\pi V_{1 m}}{4 V_{d c}}=\frac{\pi n}{4} m \\
& \cos \left(5 \theta_{1}\right)+\cos \left(5 \theta_{2}\right)+\cdots+\cos \left(5 \theta_{n}\right)=0 \\
& \cos \left(7 \theta_{1}\right)+\cos \left(7 \theta_{2}\right)+\cdots+\cos \left(7 \theta_{n}\right)=0 \\
& \cdots \cdots
\end{aligned}\right.
$$

In account that solving process is common, I take the three-level inverter for example. Assume $\mathrm{m}=0.8$, and the output voltage does not include the fifth, the seventh harmonic. The nonlinear equations are as follow,

$$
\left\{\begin{aligned}
\cos \left(\theta_{1}\right)+\cos \left(\theta_{2}\right)+\cdots+\cos \left(\theta_{n}\right)=\frac{\pi V_{1 m}}{4 V_{d c}} & =\frac{\pi n}{4} m \\
\cos \left(5 \theta_{1}\right)+\cos \left(5 \theta_{2}\right)+\cdots+\cos \left(5 \theta_{n}\right) & =0 \\
\cos \left(7 \theta_{1}\right)+\cos \left(7 \theta_{2}\right)+\cdots+\cos \left(7 \theta_{n}\right) & =0
\end{aligned}\right.
$$

Given that the homotopy algorithm is characterized by its fast convergence speed and large convergence domain, the homotopy algorithm is chosen to solve the equations.

To begin with, plug in the data:

$$
\left\{\begin{array}{c}
\cos \left(\theta_{1}\right)+\cos \left(\theta_{2}\right)+\cdots+\cos \left(\theta_{n}\right)-0.6 \pi=0 \\
\cos \left(5 \theta_{1}\right)+\cos \left(5 \theta_{2}\right)+\cdots+\cos \left(5 \theta_{n}\right)=0 \\
\cos \left(7 \theta_{1}\right)+\cos \left(7 \theta_{2}\right)+\cdots+\cos \left(7 \theta_{n}\right)=0
\end{array}\right.
$$

Regard $\theta_{i}$ as function with independent variable $t$, the derivation is:

$$
H(a, t)=F(a)+(t-1) F[a(0)]=0, t \in[0,1], a \in D
$$

Where $F_{0}(a)=F(a)-F[a(0)]$

Applying differential method, take the derivative with respect to $t$ on both side of equation:

$$
\frac{\partial H(a, t)}{\partial a} a^{\prime}(t)+\frac{\partial H(a, t)}{\partial t}=\frac{\partial F(a)}{\partial a} a^{\prime}(t)+F[a(0)]=0
$$

With arrangement, the equation is

$$
a^{\prime}(t)=-\left\{F^{\prime}[a(t)]\right\}^{-1} F[a(0)], t \in[0,1]
$$

Giving the initial value,

$$
\theta_{0}\left(\theta_{1}, \theta_{2}, \theta_{3}\right)=(0.1 \pi, 0.2 \pi, 0.3 \pi)
$$

Now, nonlineartranscendental equations become system of ordinary differential equations.four-order quaternion Runge-Kutta methodis applied to solve the equations. The formulas are as follow,

$$
\left\{\begin{array}{c}
y_{n+1}=y_{n}+\frac{h}{6}\left(k_{1}+2 k_{2}+3 k_{3}+k_{4}\right) \\
k_{1}=f\left(x_{n}, y_{n}\right) \\
k_{2}=f\left(x_{n}+\frac{h}{2}, y_{n}+\frac{h k_{2}}{2}\right) \\
k_{3}=f\left(x_{n}+\frac{h}{2}, y_{n}+\frac{h k_{3}}{2}\right) \\
k_{4}=f\left(x_{n}, y_{n}+h k_{3}\right)
\end{array}\right.
$$

Where $y_{n}$ is the parameters required; $x_{n}$ is independentvariable, here is $t$; $h$ is step length.

Set the number of iteration is $1400 h=\frac{1}{N}=1 / 1400$

With the results of each iteration as the initial value of next-time solving, I solve 5 times to improve the accuracy. 
The value of $\theta_{i} i=1,2,3$ is in table 1 . The value of $m$ is also calculated, as well as its error.

Table 1 Results of the equations

\begin{tabular}{|c|c|c|c|c|c|c|}
\hline \multicolumn{3}{|c|}{ The initial value (rad) } & \multicolumn{3}{|c|}{ Results $(\mathrm{rad})$} & $\begin{array}{c}\text { Error of } m \\
(\%)\end{array}$ \\
\hline$\theta_{1}$ & $\theta_{2}$ & $\theta_{3}$ & $\theta_{1}$ & $\theta_{2}$ & $\theta_{3}$ & \\
\hline 0.3141 & 0.6283 & 0.9425 & 0.4613 & 0.8870 & 1.1209 & 4.09 \\
0.4613 & 0.8870 & 1.1209 & 0.5016 & 0.9397 & 1.1253 & 0.2 \\
0.5016 & 0.9397 & 1.1253 & 0.5088 & 0.9484 & 1.1254 & 0.06 \\
0.5102 & 0.9501 & 1.1255 & 0.5100 & 0.9498 & 1.1255 & 0.01145 \\
0.5088 & 0.9484 & 1.1254 & 0.5102 & 0.9501 & 1.1255 & $5.83 \times 10^{-4}$ \\
\hline
\end{tabular}

As table 1 shown, the algorithm can meet the requirement of accuracy. The amplitude of the fifth and the seventh harmonics is

$$
\left\{\begin{array}{l}
V_{5}=\frac{4 V_{d c}}{5 \pi}\left[\cos \left(5 \theta_{1}\right)+\cos \left(5 \theta_{2}\right)+\cos \left(5 \theta_{3}\right)\right] \\
V_{7}=\frac{4 V_{d c}}{7 \pi}\left[\cos \left(7 \theta_{1}\right)+\cos \left(7 \theta_{2}\right)+\cos \left(7 \theta_{3}\right)\right]
\end{array}\right.
$$

Derives

$$
\left\{\begin{array}{c}
V_{5}=1.2127 \times 10^{-4} \ll 0 \\
V_{7}=-3.3876 \times 10^{-4} \ll 0
\end{array}\right.
$$

In this way, we can think the fifth and the seventh harmonics is eliminated. In this way, the value of $\theta_{1}, \theta_{2}, \theta_{3}$ satisfies the requirement. The solution is,

$$
\left\{\begin{array}{l}
\theta_{1}=0.5102 \\
\theta_{2}=0.9501 \\
\theta_{3}=1.1255
\end{array}\right.
$$

With selected harmonic elimination pulse width modulation, the wave quality of output voltage can be described with $T H D$ (Total Harmonics Distortion) as follow,

$$
T H D=\sqrt{\frac{\sum_{\substack{i=6 k \pm 1 \\ k=1,2,3 \cdots}}^{\infty} V_{i}^{2}}{V_{1}^{2}}} \times 100=\sqrt{\frac{V_{5}^{2}+V_{7}^{2}+V_{11}^{2}+\cdots V_{43}^{2}+\cdots}{V_{1}^{2}}} \times 100 \%
$$

Where

$$
V_{n}=\frac{4 V_{d c}}{n \pi}\left[\cos \left(n \theta_{1}\right)+\cos \left(n \theta_{2}\right)+\cos \left(n \theta_{3}\right)\right]
$$

With the help of Matlab, the corresponding THDis,

$$
T H D=11.55 \%
$$

\section{Evaluation and Optimization of the Model}

Thehomotopy algorithm has many advantages when solving nonlinear equations such as its fast convergence speed and large convergence domain. And also, thehomotopy algorithm has less computational effort and high probability of success. Nevertheless, the accuracy of thehomotopy algorithm is lower. To get accurate answer, iteration works must be done.

Given the homotopy algorithm has large convergence domain, fast convergence speed but low accuracy while Newton method has high accuracy but small convergence domain, optimal model combine the two methods. The concrete steps are as follow: 
To begin with, assume reasonable initial value and do a fixed number of iterations with the homotopy algorithm. Then have the results as the initial value of Newton method to iterate. Given the results of the homotopy algorithm is close to accurate results, Newton's method convergent inevitably. With the optimized model, the results are

$$
\left\{\begin{array}{l}
\theta_{1}=0.5103 \\
\theta_{2}=0.9501 \\
\theta_{3}=1.1255
\end{array}\right.
$$

\section{References}

[1] LIZhidian, A Novel Algorithm for Real-Time Solution of Nonlinear Surmount SHET Equations.

[2] YE Manyuan, Study of Selected Harmonics Elimination Control Technology of Multi-level Inverter, School of Electrical and Eletronic Engineering, East China JiaotongUniverrsity.

[3]SHI Yuan, Research of Mathematical Model and Switching Angle's Computational Method of Inverter with SHEPWM.

[4]XIE Yunxiang, Homotopy Model and Its Algorithm Analysis of the Inverter Harmonic Elimination Control Strategy, College of Electrical Engineering, South China University of Technology, Guangzhou 510640, China

[5]ZHOU Lian, Homotopy-Based Computation for Harmonic Elimination in PWM Inverters

[6]FEI Wangmin, Inclusion Relationship of the Solutions of SHEPWM Tec, Nanjing Normal University

[7]SUN Yichao, Control Strategy of DC Voltage Balance and Power Equilibrium for Grid-connected Cascaded H-bridge Converters, Electric PowerAutomation Equipment, 2014.34(1) 